\title{
Effects of Various Geometric Designs on the Flow Characteristics of a Triangular Rotary Engine
}

\author{
Chiu-Fan Hsieh ${ }^{1} \&$ Hao-Yu Cheng ${ }^{1}$ \\ ${ }^{1}$ Department of Mechanical and Computer-Aided Engineering, National Formosa University, Taiwan \\ Correspondence: Chiu-Fan Hsieh, Department of Mechanical and Computer-Aided Engineering, National \\ Formosa University, 64 Wunhua Road, Huwei, Yunlin, Taiwan. E-mail: cfhsieh@nfu.edu.tw
}

Received: December 5, 2014 Accepted: December 28, 2014 Online Published: January 5, 2015

doi:10.5539/mer.v5n1p1

URL: http://dx.doi.org/10.5539/mer.v5n1p1

\begin{abstract}
Although rotary engines can potentially be used as hydrogen fuel engines, the contact condition between the casing and the apex seal on the triangular rotor impacts sealing performance, which then directly affects leakage problems. In this paper, therefore, a rotary engine's internal gas flow characteristics are investigated by using the CFD package FLUENT to construct a fluid analysis model. For comparative convenience, assuming that the simulated gas is air, and then, using a set circular radius outside the casing, analyze three different cases with various geometric design parameters (the $K$ factor) and their effects on the internal pressure, streamlining, and leakage. The results indicate that the $K$ factor design produces different rotor profiles with different working chamber volumes: the lower the $K$ factor, the larger the working chamber volume. Nevertheless, although this design can improve the combustion and compression efficiencies, it may lead to increased internal pressure variation and raised pressure. In addition, when the clearance is small, it may result in a larger leakage problem, negatively affecting rotary engine performance.
\end{abstract}

Keywords: rotary engine, sealing property, leakage, flow characteristics, CFD

\section{Introduction}

The Wankel engine, commonly called a rotary engine, is a type of internal combustion engine that instead of using reciprocating pistons uses an eccentric rotary design to convert pressure into a rotating motion. In addition to apex seals, an eccentric shaft, and sun gears, the rotary engine consists of two important parts: a specially designed chamber and a triangular rotor. The movement of this latter is such that its tips are always in contact with the chamber walls so that at any instant, the chamber is divided into three regions. Each of these three areas houses a different part of the combustion process, so that each of the rotary engine's four different jobs - intake, compression, combustion, and exhaust - occurs in its own section of the housing. It is thus equivalent to having a dedicated cylinder for each of the four jobs, with the piston moving continually from one to the next.

The relevant research on rotary drives includes an early study of trochoid envelopes, which outlined a planetary motion that can be applied in engines or pumps (Colbourne, 1974). A more recent paper proposed a very general mathematical relation for a generating arc traveling on a hypotrochoidal path and then compared the flow rate and pocket displacement (Beard, Hall, \& Soedel, 1991). Subsequent work then developed kinematic equations and performed a dynamic force analysis of the apex seal on the rotary drive (Beard \& Pennock, 1997). Other kinematic analyses of the epicycloidal and hypocycloidal mechanisms for a rotary engine identified differences in their compression ratios and transmission angle (Shih, 1993) and the influence of flowrate irregularity, compression ratio, and pressure angle using various geometric design parameters (Bonandrini, Mimmi, \& Rottenbacher, 2009). Recently, a new method for deriving the deviation function was developed for designing a rotary engine with apex seal profiles that can be arc or non-arc curves (Rose \& Yang, 2013, 2014).

For the use of hydrogen fuel in the Wankel engine, related studies can be traced back to Mazda in the 1990s, since when several drawbacks of hydrogen engines have been identified (Salanki \& Wallace James, 1996). A very recent study, however, shows that adding hydrogen to gasoline can improve both thermal efficiency and power output (Amrouche et al., 2014), meaning that the rotary engine has the potential to be a "green" hydrogen fuel engine. Nevertheless, if this goal is to be realized, many problems remain to be solved, including the rotary engine's combustion efficiency and the strength and sealing properties of its rotor. It is for this reason that the rotary engine is not currently prevalent in industry. 
Because the key to the rotary engine's performance is geometric design, in this paper, we investigate the flow characteristics of various geometric designs for the rotary engine by developing a fluid analysis model of a triangular rotary engine. The following sections outline the mathematical model of the casing profile and triangular rotor and then discuss fluid modeling and the flow characteristics inside the rotary engine.

\section{Mathematical Model of the Rotary Engine Geometry}

The theoretical curve of the rotary engine housing is most commonly epitrochoidal, as shown in Figure 1. Assuming that the rolling circle $\Sigma_{r}$ with radius $\rho_{r}$ follows another fixed circle $\Sigma_{b}$ with radius $\rho_{b}$ rolling by an internal tangent, then when $\rho_{r}>\rho_{b}, \rho_{r}=1.5 \rho_{b}$, and $\alpha=3 \beta$, the housing curve is formed by the movement trajectory of point $p$. The equation can then be represented as

$$
\mathbf{r}_{1}=\left[\begin{array}{lll}
x_{1} & y_{1} & 1
\end{array}\right]^{T}
$$

where

$$
\begin{aligned}
& x_{1}=c \cos \alpha+\frac{3 K c^{2}}{\rho_{r}} \cos \beta \\
& y_{1}=c \sin \alpha+\frac{3 K c^{2}}{\rho_{r}} \cos \beta
\end{aligned}
$$

Here, $c$ is the eccentric distance, $K$ is defined as $\ell / c . x_{1}$ and $y_{1}$ are the coordinate positions of $p, \alpha$ is the revolution angle of the rolling circle $\Sigma_{r}$ around the fixed circle $\Sigma_{b}$, and $\beta$ is the revolution angle of the rolling circle $\Sigma_{r}$ around the its own axis.

Based on the operational principle of the rotary engine, the apexes of the triangular rotor should always stay in contact with the housing, and the triangular rotor and housing should never collide during operation. Hence, to obtain the triangular rotor profile, circle $\Sigma_{r}$ should be fixed (see Figure 1). Specifically, circle $\Sigma_{b}$ rotates along circle $\Sigma_{r}$ and then forms a series of housing curves with circle $\Sigma_{b}$. The inner envelope of the housing curves is the triangular rotor profile. According to the condition of a common tangent on the geometrical contact point, the equation of the triangular rotor curve can be derived as follows:

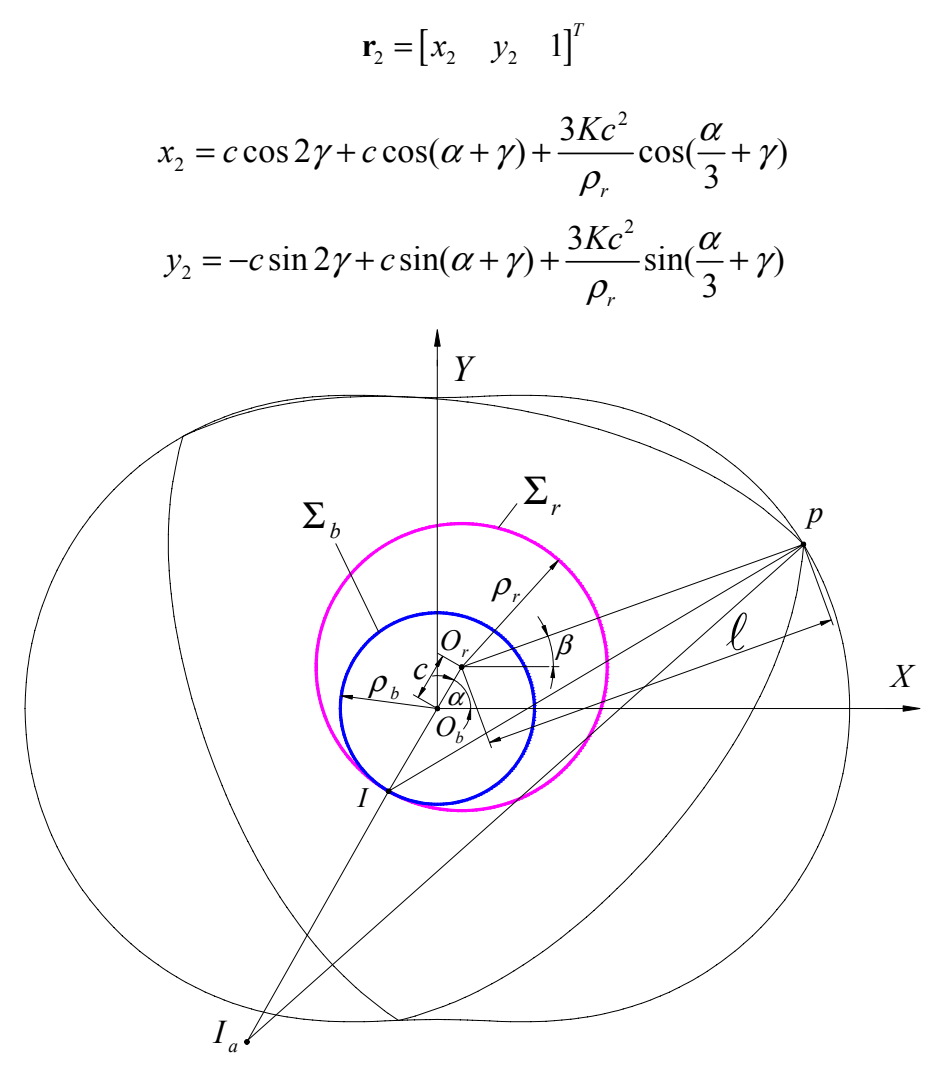

Figure 1. Generation principle of the profile 
The contact point can then be obtained by considering

and then

$$
f=\frac{\partial x_{2}}{\partial \alpha} \cdot \frac{\partial y_{2}}{\partial \gamma}-\frac{\partial x_{2}}{\partial \gamma} \cdot \frac{\partial y_{2}}{\partial \alpha}=0
$$

$$
f=-\sin \frac{2}{3} \alpha+\frac{K c}{\rho_{r}} \sin (\alpha+3 \gamma)+\sin \left(\frac{1}{3} \alpha+3 \gamma\right)=0
$$

An actual rotary engine structure, however, contains apex seals whose arc radius is $e$ and whose center is the peak of the triangular rotor. Hence, the actual housing profile must be offset outward by distance $e$, as represented by

$$
\mathbf{R}_{1}=\left[\begin{array}{lll}
x_{1}+n_{x 1} e & y_{1}+n_{y 1} e & 1
\end{array}\right]^{T}
$$

where

$$
\begin{gathered}
\mathbf{n}_{1}=\frac{\frac{\partial \mathbf{r}_{1}}{\partial \alpha} \times \mathbf{k}}{\left|\frac{\partial \mathbf{r}_{1}}{\partial \alpha}\right|}=\frac{\left[\begin{array}{lll}
t_{y 1} & -t_{x 1} & 0
\end{array}\right]^{T}}{\sqrt{t_{x 1}^{2}+t_{y 1}^{2}}}=\left[\begin{array}{c}
n_{x 1} \\
n_{y 1} \\
0
\end{array}\right] \\
\frac{\partial \mathbf{r}_{1}}{\partial \alpha}=\left[\begin{array}{c}
t_{x 1} \\
t_{y 1} \\
0
\end{array}\right]=\left[\begin{array}{c}
-\frac{K c^{2}}{\rho_{r}}\left(\frac{K c}{\rho_{r}} \sin \alpha+\sin \frac{\alpha}{3}\right) \\
\frac{K c^{2}}{\rho_{r}}\left(\frac{K c}{\rho_{r}} \cos \alpha+\cos \frac{\alpha}{3}\right) \\
0
\end{array}\right]
\end{gathered}
$$

The actual rotor profile equation can then be expressed as

$$
\mathbf{R}_{2}=\left[\begin{array}{lll}
x_{2}+n_{x 2} e & y_{2}+n_{y 2} e & 1
\end{array}\right]^{T}
$$

where

$$
\begin{gathered}
\mathbf{n}_{2}=\frac{\frac{\partial \mathbf{r}_{2}}{\partial \alpha} \times \mathbf{k}}{\left|\frac{\partial \mathbf{r}_{2}}{\partial \alpha}\right|}=\frac{\left[\begin{array}{lll}
t_{y 2} & -t_{x 2} & 0
\end{array}\right]^{T}}{\sqrt{t_{x 2}{ }^{2}+t_{y 2}{ }^{2}}}=\left[\begin{array}{c}
n_{x 2} \\
n_{y 2} \\
0
\end{array}\right] \\
\frac{\partial \mathbf{r}_{2}}{\partial \alpha}=\left[\begin{array}{c}
t_{x 2} \\
t_{y 2} \\
0
\end{array}\right]=\left[\begin{array}{c}
-\frac{K c^{2}}{\rho_{r}}\left[\left(1+3 \gamma^{\prime}\right) \sin \left(\frac{\alpha}{3}+\gamma\right)+\left(\frac{\rho_{r}}{K c}+\frac{\rho_{r}}{K c} \gamma^{\prime}\right) \sin (\alpha+\gamma)+2 \frac{\rho_{r}}{K c} \gamma^{\prime} \sin 2 \gamma\right] \\
\frac{K c^{2}}{\rho_{r}}\left[\left(1+3 \gamma^{\prime}\right) \cos \left(\frac{\alpha}{3}+\gamma\right)+\left(\frac{\rho_{r}}{K c}+\frac{\rho_{r}}{K c} \gamma^{\prime}\right) \cos (\alpha+\gamma)-2 \frac{\rho_{r}}{K c} \gamma^{\prime} \cos 2 \gamma\right]
\end{array}\right] \\
\gamma^{\prime}=-\frac{1}{9}\left[\begin{array}{c}
2 K c\left(\cos \frac{2}{3} \alpha-\frac{\rho_{r}}{K c} \cos (\alpha+3 \gamma)\right. \\
\left.1-\frac{\rho_{r} \cos (\alpha+3 \gamma)+\cos \left(\frac{1}{3} \alpha+3 \gamma\right)}{3}\right]
\end{array}\right]
\end{gathered}
$$

For comparative convenience, when assessing the various rotor and housing profile designs and their effects on a rotary engine's flow characteristics, we assume a set circular radius outside the casing of $92.75 \mathrm{~mm}$. Table 1 shows the design parameters for the three cases, including the three $\mathrm{K}$ factors used to explain inter-design differences. Figure 2 shows the design results for the rotary engine: when the $\mathrm{K}$ factor is small, the working chamber volume between the casing and the triangle rotor is large. 
Table 1. Parameter values (Unit: $\mathrm{mm}$ )

\begin{tabular}{ccc}
\hline & $e$ & $\ell$ \\
\hline $\mathrm{K}=10($ Case 1) & 8.432 & 84.318 \\
$\mathrm{~K}=7.5($ Case 2$)$ & 10.912 & 81.838 \\
$\mathrm{~K}=6$ (Case 3) & 13.25 & 79.5 \\
\hline
\end{tabular}

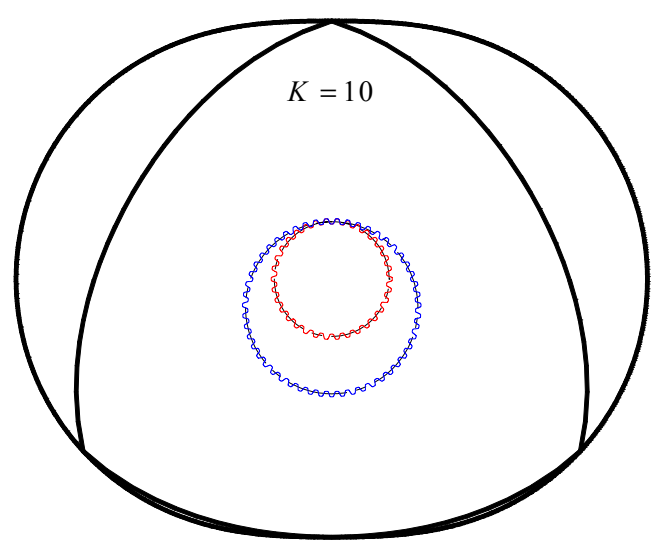

(a) $\mathrm{K}=10$

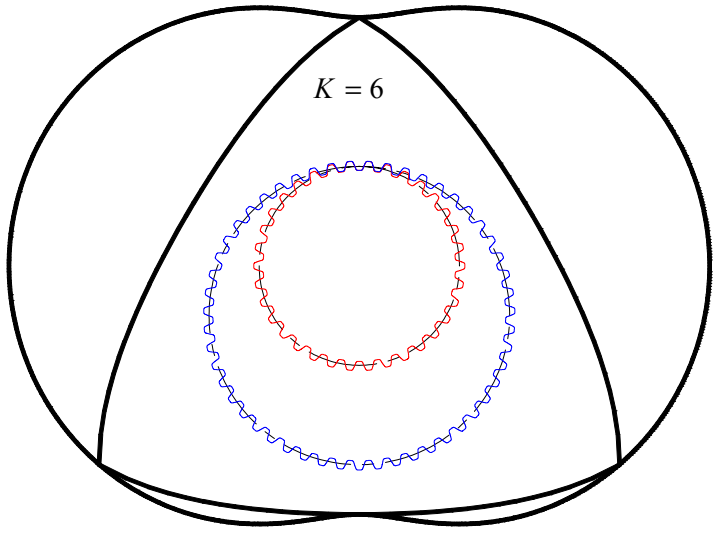

(c) $\mathrm{K}=6$

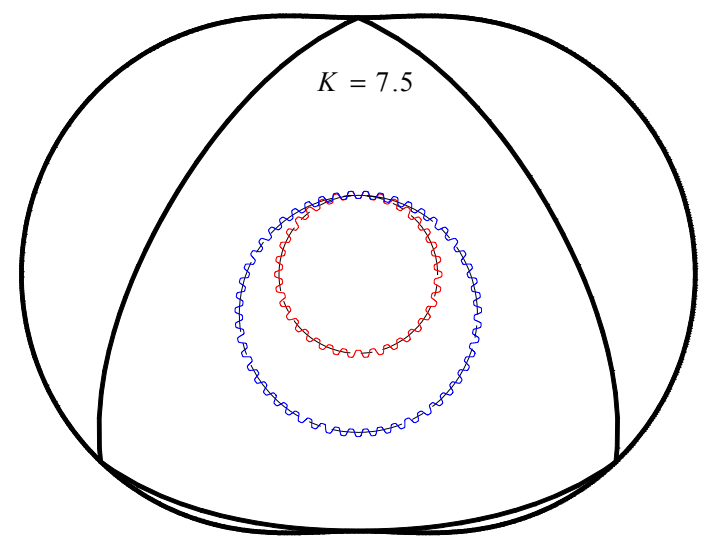

(b) $\mathrm{K}=7.5$

$$
\begin{array}{r}
K=10 \\
K=7.5
\end{array}
$$$$
K=6
$$

(d) Casing profiles

Figure 2. Design results for the rotary engine

\section{Fluid Analysis and Discussion}

\subsection{Governing Equations}

The CFD (Computational Fluid Dynamics) package FLUENT used in this study solves conservation equations of mass and momentum using a finite volume approach. The continuity equation can be expressed as

$$
\frac{\partial \rho}{\partial t}+\nabla \cdot \rho \vec{V}=0
$$

The momentum equation is then

$$
\rho\left[\frac{\partial(\vec{V})}{\partial t}+(\nabla \cdot \vec{V}) \vec{V}\right] \nabla p+(\nabla \tilde{\tau})=\rho \vec{g}+\vec{f}
$$

where $p$ is the static pressure, $\tilde{\tau}$ is the stress tensor, and $\rho \vec{g}$ and $\vec{f}$ are the gravitational body force and surface forces, respectively.

Turbulence is accounted for using a standard $k$ - $\varepsilon$ two-equation model, chosen because its convergence is better than any other model type with no appreciable difference in flow structure: 


$$
\begin{gathered}
\frac{\partial}{\partial t}(\rho k)+\nabla \cdot(\rho k V)=\nabla \cdot\left[\left(\mu+\frac{\mu_{t}}{\sigma_{k}}\right) \nabla k\right]+G_{k}+G_{b}-\rho \varepsilon-y_{m}+S_{k} \\
\frac{\partial}{\partial t}(\rho \varepsilon)+\nabla \cdot(\rho \varepsilon V)=\nabla \cdot\left[\left(\mu+\frac{\mu_{t}}{\sigma_{\varepsilon}}\right) \nabla \varepsilon\right]+C_{1 \varepsilon} \frac{\varepsilon}{k}\left(G_{k}+C_{3 \varepsilon} G_{b}\right)-C_{2 \varepsilon} \frac{\varepsilon^{2}}{k}+S_{\varepsilon}
\end{gathered}
$$

Here, $G_{k}$ represents the generation of turbulent kinetic energy due to the mean velocity gradients, $G_{b}$ is the generation of turbulent kinetic energy due to buoyancy, and $y_{m}$ represents the contribution of the fluctuating dilatation in compressible turbulent flow to the overall dissipation rate. $C_{1 \varepsilon}, C_{2 \varepsilon}$, and $C_{3 \varepsilon}$ are constants, $\sigma_{k}$ and $\sigma_{\varepsilon}$ are the turbulent Prandtl numbers for $k$ and $\varepsilon$, respectively, and $S_{k}$ and $S_{\varepsilon}$ are user-defined source terms.

\subsection{Assumptions and Boundary Conditions}

For simulative convenience, the fluid used in this research is air (density is $1.293 \mathrm{~kg} / \mathrm{m}^{3}$ and viscosity is $1.81 \mathrm{E}-05 \mathrm{~kg} / \mathrm{m}-\mathrm{s})$. The fluid analysis also makes the following important assumptions:

(1) The fluid is analyzed using a turbulent model ( $k-\varepsilon$ model).

(2) Flow is unsteady, compressible and turbulent.

(3) Conjugate heat transfer and radiation effects are neglected.

(4) The fluid is initially stationary; the flow is two-dimensional.

The input conditions are as follows: inlet pressure $=$ two atmospheric pressure $(202,650 \mathrm{~Pa})$, outlet pressure $=$ one atmospheric pressure $(101,325 \mathrm{~Pa})$, shaft speed $=1800 \mathrm{rpm}$.

During operation, the effect on the rotary engine of imbalance gas pressure and rotation inertia or centrifugal force changes the contact force on the apex seal causing it to move, which leaves a tiny clearance between the apex seal and the housing surface that can lead to leakage problems. Hence, for simulative convenience, we assume a set clearance of $0.15 \mathrm{~mm}$ in each case and then compare the leakage condition in various designs.

\subsection{Grid Generation}

Taking the case of $\mathrm{K}=6$ as an example, the fluid model of the rotary engine is in $2 \mathrm{D}$ and has four regions: the inlet, outlet, rotor, and casing. The rotor's swaying motion, which changes the volumetric displacement between rotor and casing, can be discretized using an unstructured grid (e.g., triangular cell) generated by the FLUENT GAMBIT package. The resulting mesh in the case of $\mathrm{K}=6$ is shown in Figure 3. This simulation includes 6 simulation cycles of the rotation shaft with a total of 20,000 steps, each counted as $1 \mathrm{E}-05 \mathrm{sec}$. In the dynamic grid model, the internal pressure in the interval $0.01 \sim 0.05 \mathrm{~s}$ is calculated as in Figure 4. As Figure 4(a) shows, the volume is largest on the right side, with the apex seal separated from the inlet, which is ready for compression. In Figure 4(b), the volume is lessening and starting to compress, and the internal pressure rises to about 7 atmospheric pressures. Figure 4(c) then shows the condition after compression, when the pressure drops off because of leakage; Figure 4(d) illustrates the depression after compression, when volume gradually increases; and Figure 4(e) depicts the major depression for exhausting gas to the outlet.

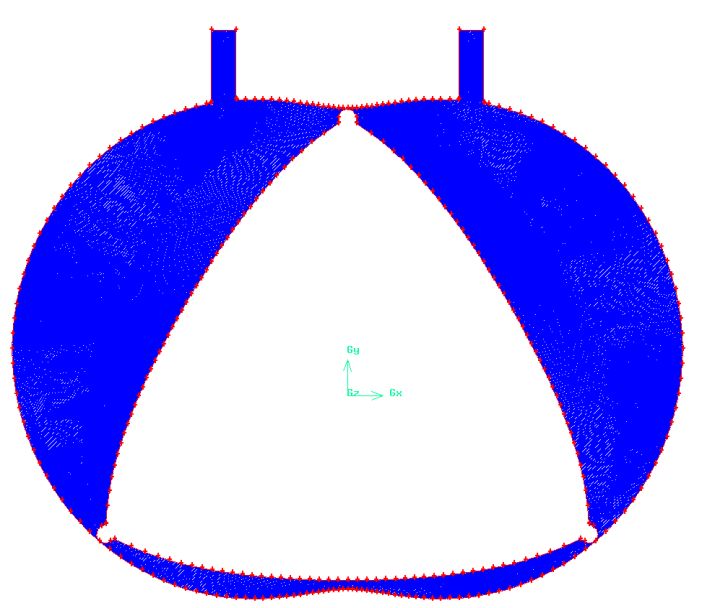

(a) Whole mesh

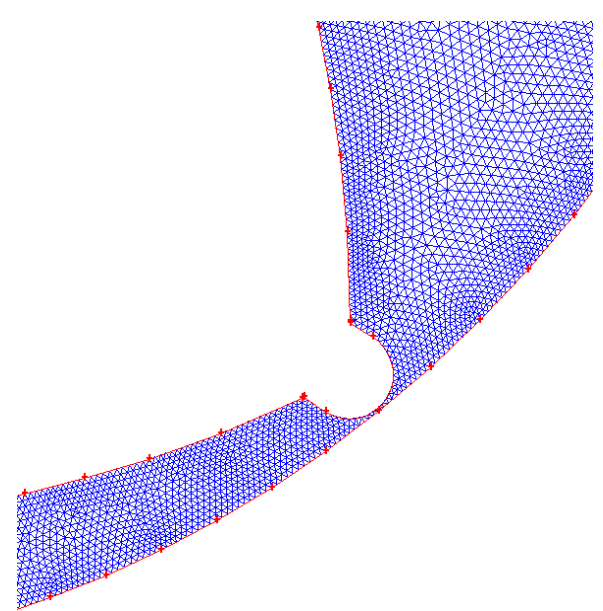

(b) Mesh at the apex seal

Figure 3. Resulting mesh 


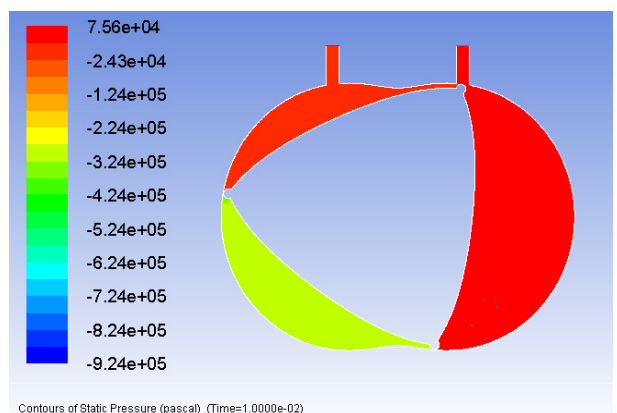

(a) $0.01 \mathrm{~s}$

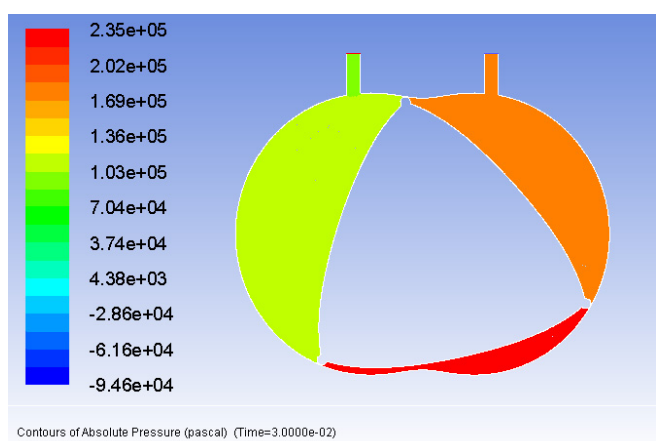

(c) $0.03 \mathrm{~s}$

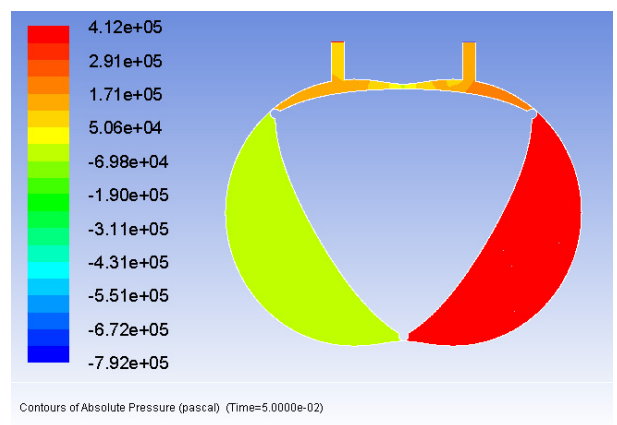

(e) $0.05 \mathrm{~s}$

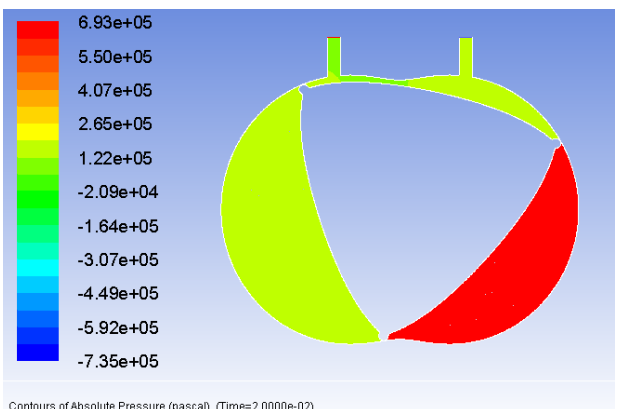

(b) $0.02 \mathrm{~s}$

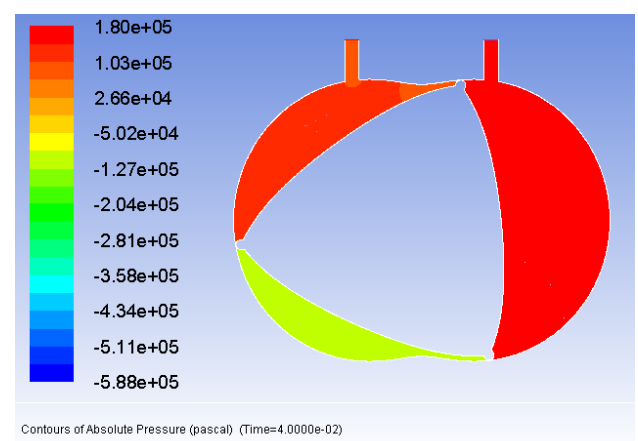

(d) $0.04 \mathrm{~s}$

Figure 4. Pressure calculation for the rotary engine

Based on the calculation mode used in this paper, the grid information for the three cases is as shown in Table 2.

Table 2. Grid information

\begin{tabular}{cccc}
\hline & Cells & Faces & Nodes \\
\hline Case $1(K=10)$ & 193,386 & 291,957 & 98,571 \\
Case 2 $(K=7.5)$ & 237,668 & 358,328 & 120,660 \\
Case 3 $(K=6)$ & 275,812 & 415,494 & 139,682 \\
\hline
\end{tabular}

\subsection{CFD Results and Discussion}

The working chamber volume of the rotary engine affects both compression efficiency and the fluid flow characteristics. For example, when $K$ equals 6, Case 3 has the largest volume, followed by Case 2 and then Case 1. Setting particular survey points in the three cases (see Figure 5) throws more light on the effect of different rotary engine geometric designs on the internal flow field, particularly flow variations and leakage in the rotor. The CFD results reported here are based on one revolution of the triangular rotor. 


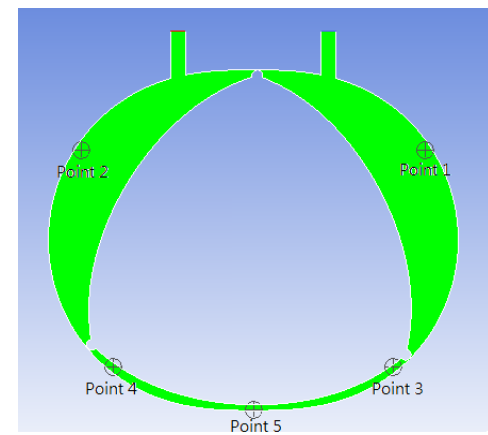

(a) Case 1

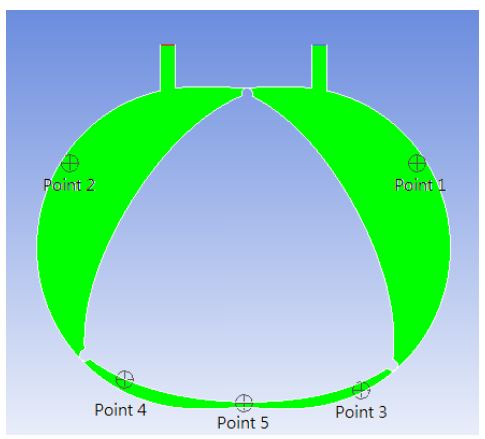

(b) Case 2

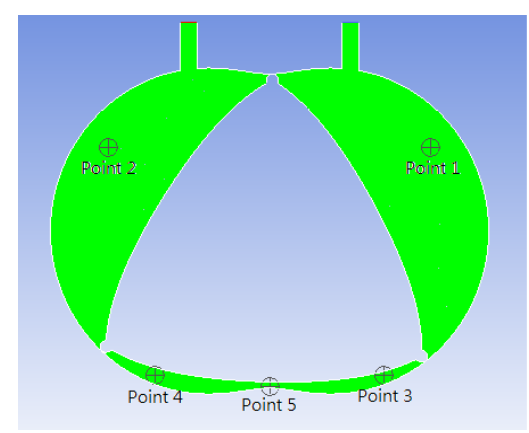

(c) Case 3

Figure 5. Survey points in the three cases

Because the survey points are covered during rotor operation, their pressure curves may be subject to a discontinuous phenomenon that leads to missing data. Even so, it is still possible to calculate most pressure values and analyze the differences. The pressure results for points 1 and 2 (see Figure 6) show no obvious differences among the three cases. Hence, near the inlet and outlet port, the rotor profile design has no obvious influence. Entering the compression area at point 3, however, there is a significant rise in pressure as the pressure curve changes with variation in the working chamber volume (Figure 7). The larger the change in volume, the greater the pressure variation, with the largest change occurring in Case 3 (Figure 7(a)). Nearing the compression area at point 4 , the larger working chamber volume produces more obvious compression and an increased pressure (Figure 7(b)). At point 5, which is located in the position of being ready to leave the compression, all three cases have significant depression (Figure 7(c)).

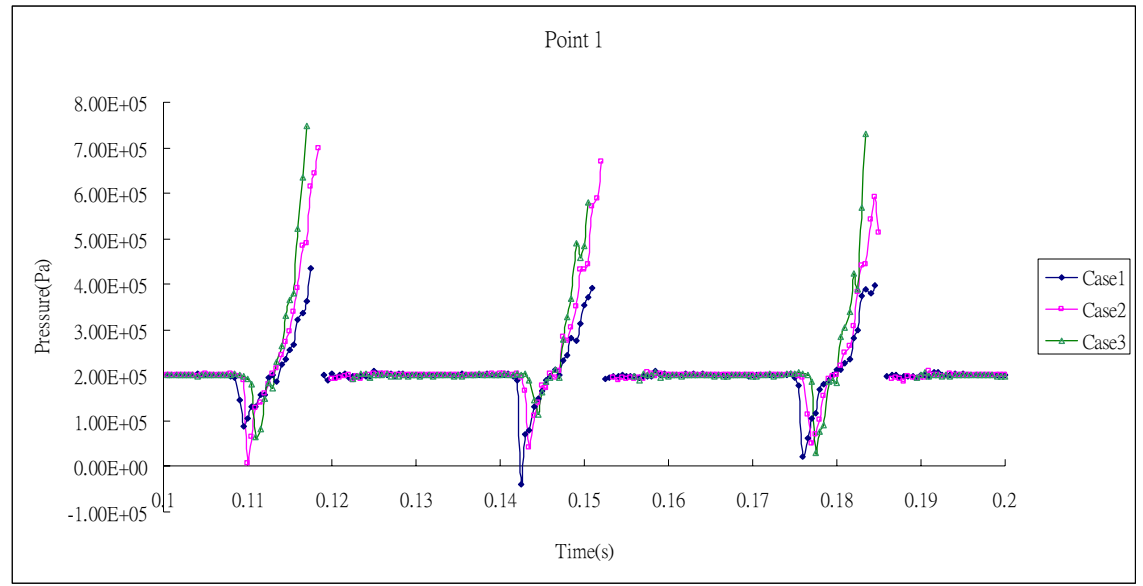

(a) Point 1

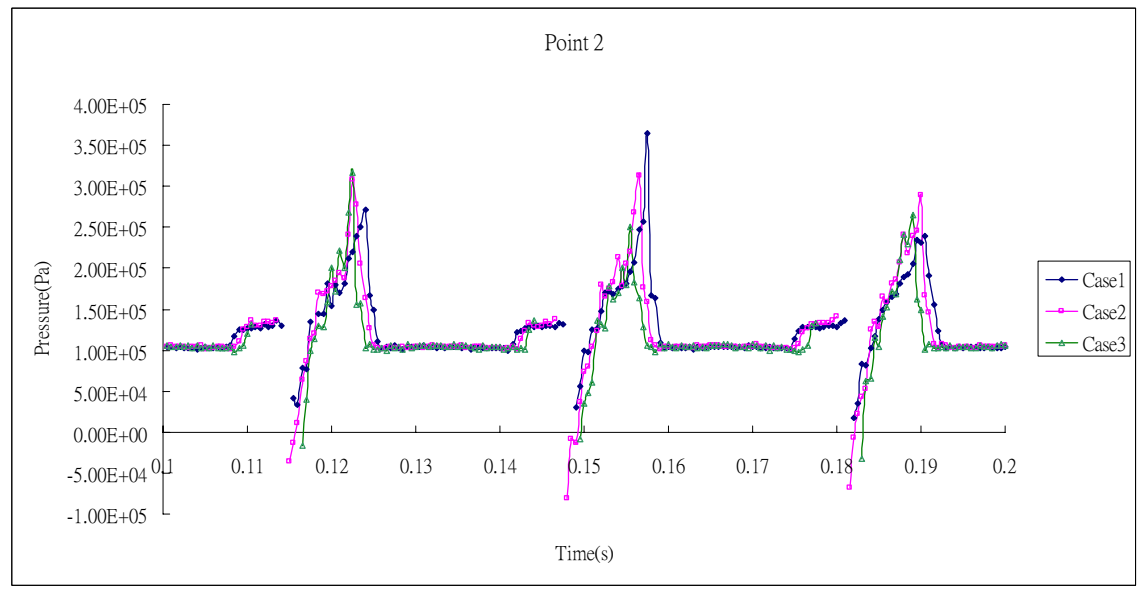

(b) Point 2

Figure 6. Pressure on points 1 and 2 
No matter the case, from point 3 to point 5 , the pressure increases during compression but at the moment of leaving the compression area, all pressure lowers, even the larger negative pressure. During the process, Case 3 has the largest positive and negative pressure peaks, so when $\mathrm{K}$ is small, it has a larger working chamber volume. Overall, the rotary engine produces large volume changes during operation, which enlarges internal pressure differences and leads to better compression efficiency. Nevertheless, there may also be larger pressure fluctuation, which could lower engine stability and affect power performance.

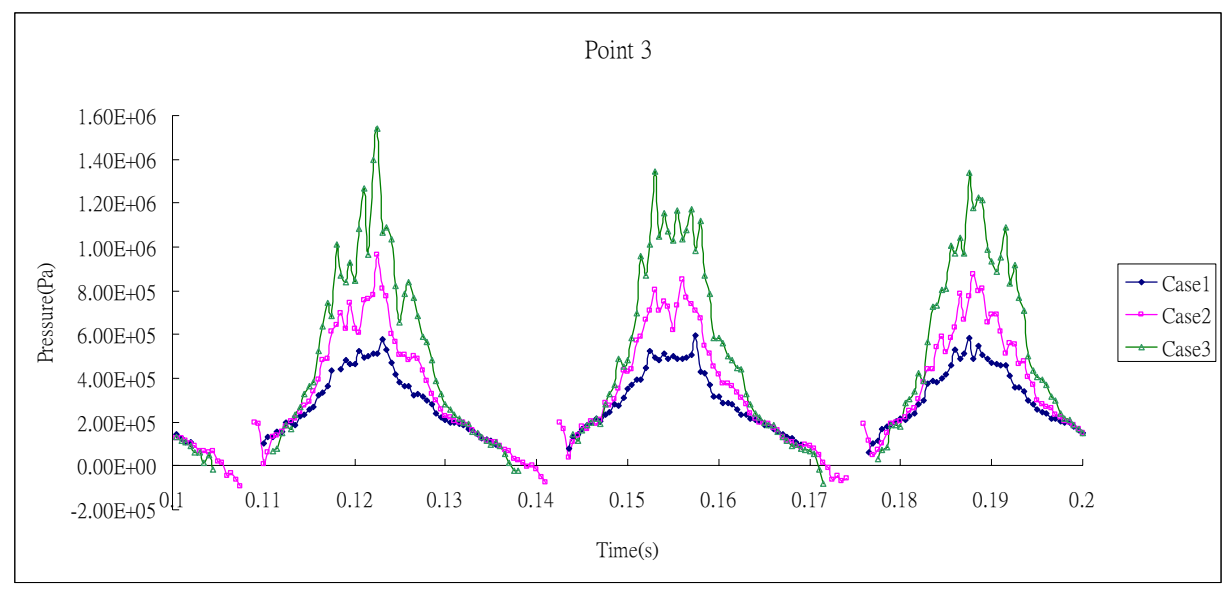

(a) Point 3

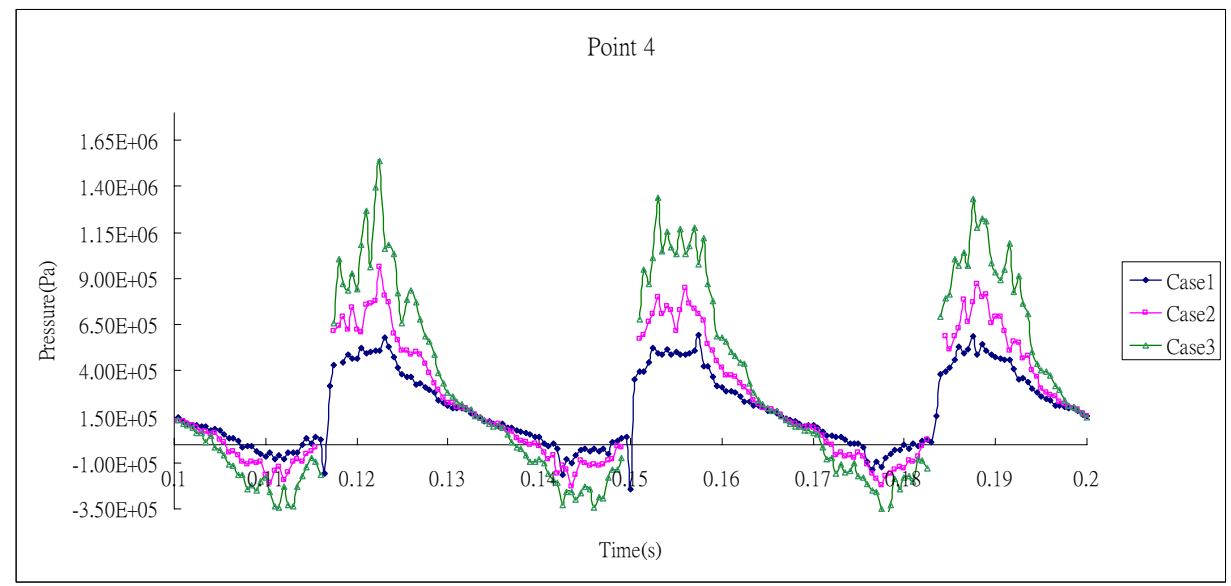

(b) Point 4

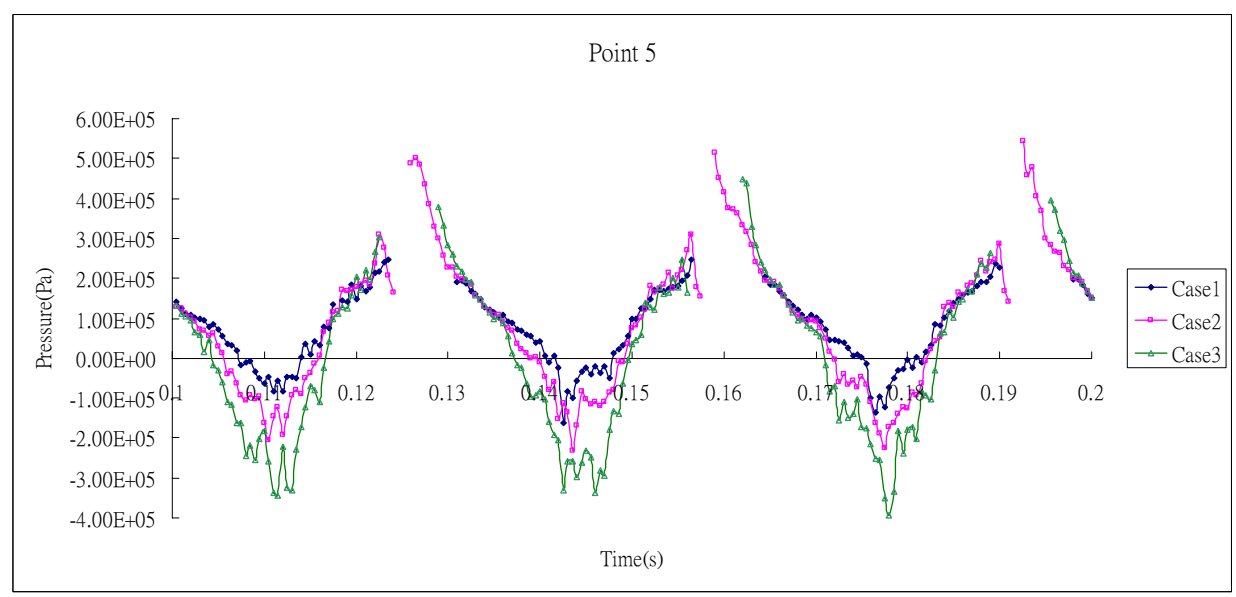

(c) Point 5

Figure 7. Pressure on points $3-5$ 
As regards variation in the inlet and outlet pressures, it is affected by changes in the working chamber volume, which is at its largest as gas moves into the chamber from the inlet. After that, the inlet pressure decreases because of the $K$ factor: the smaller the $K$ factor, the larger the working chamber volume, meaning a large pressure drop in the inlet port. The magnitude of this pressure fluctuation in the inlet port ranks as follows (see Figure 8): Case $3>$ Case $2>$ Case 1 . Changes in the working chamber volume during operation also affect the outlet pressure in the low pressure area, resulting in pressure drops and fluctuations in certain positions. A comparison of the outlet pressure fluctuations among the three cases, however, shows no significant differences. The results for the inlet and outlet ports suggest that the $K$ value is a key factor for the inlet port, one that causes large fluctuations in the inlet pressure. Specifically, the smaller the $K$ value, the greater the fluctuation.

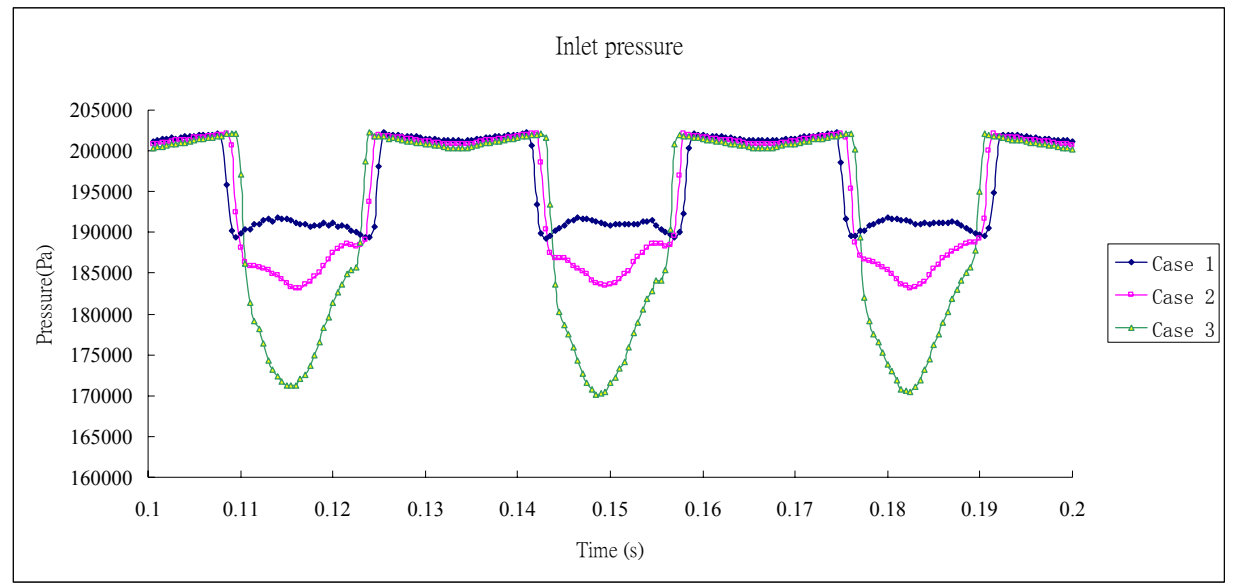

Figure 8. Pressure at the inlet

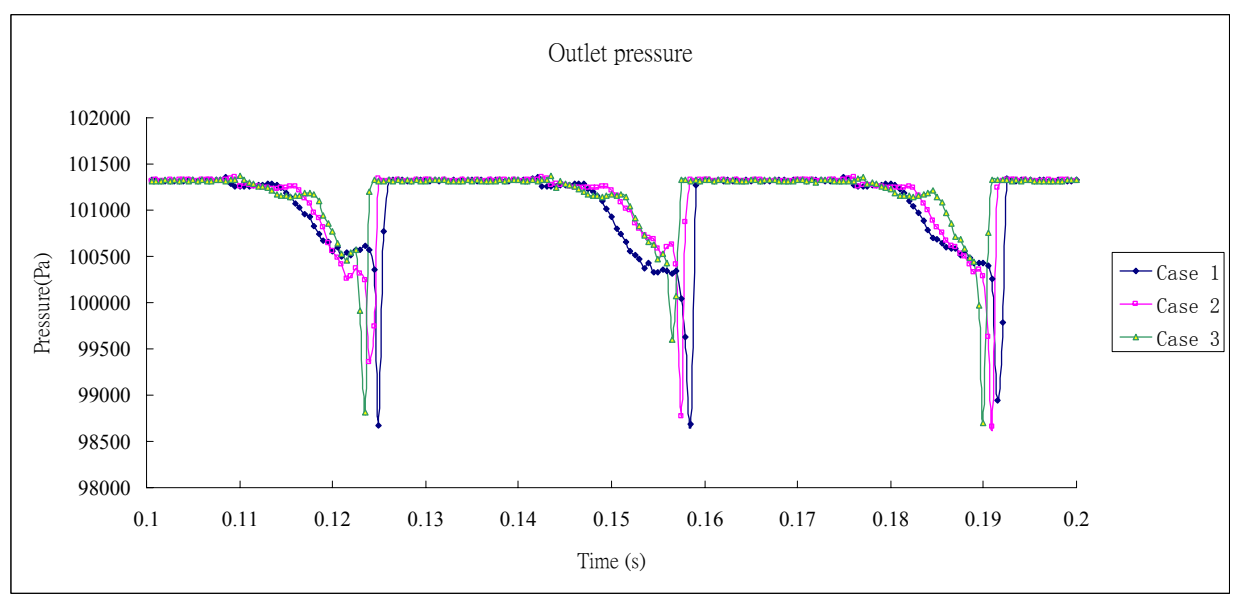

Figure 9. Pressure at the outlet

Streamlining of the three cases also reveals that when gas enters the inlet port, a vertex occurs between the casing and rotor (see Figure 10). These turbulence flows are also likely to affect the rotary engine's leakage level.

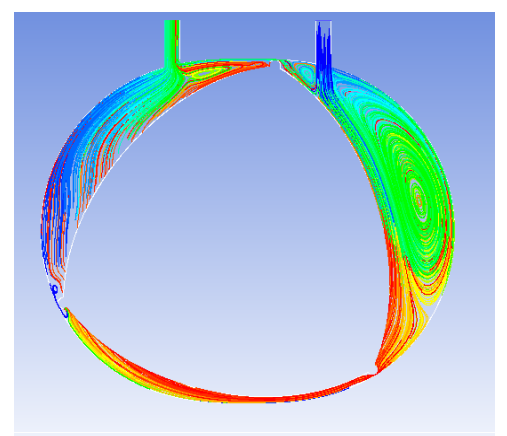

(a) Case 1

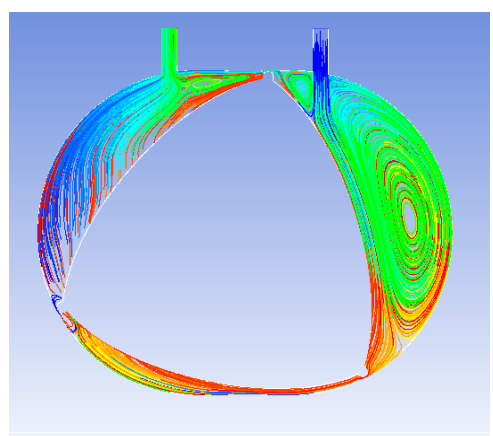

(b) Case 2

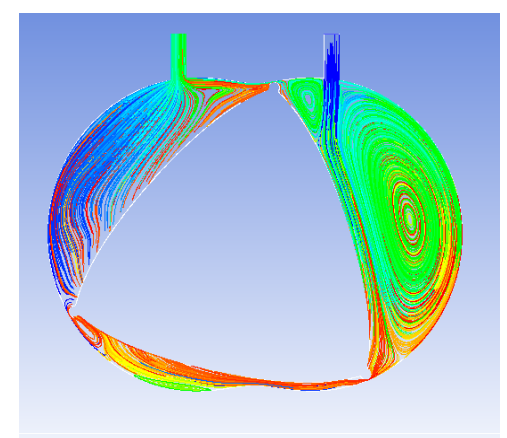

(c) Case 3

Figure 10. The three cases streamlined 
Assuming the existence of a tiny clearance between the casing and apex seal, the leakage situation is represented by streamlining the flow velocity of all three cases (see Figure 11), which demonstrates that fluid flows from the high pressure area to the low pressure area. Figure 11(a) illustrates the situation in Case 1, in which the leakage position is concentrated in the clearance, with a flow velocity up to $189 \mathrm{~m} / \mathrm{s}$ compared to $218 \mathrm{~m} / \mathrm{s}$ in Case 2 (Fig, 11(b)). The most severe leakage occurs in Case 3 (Figure 11(c)), with a flow velocity of up to $293 \mathrm{~m} / \mathrm{s}$. These observations indicate that when internal pressure rises, the leakage level increases: the highest internal pressure in Case 3 results in both the largest pressure difference and the greatest leakage magnitude. The analysis thus clearly demonstrates that the design of the rotary engine profile directly affects leakage during engine operation and has a significant effect on engine performance.

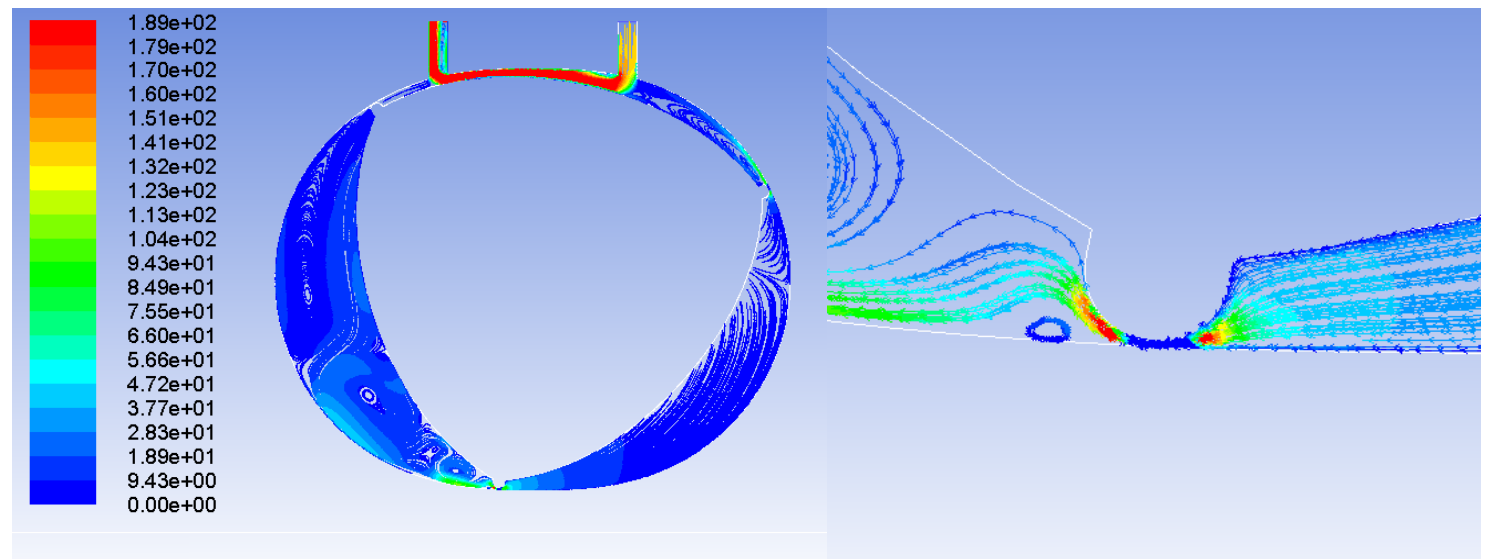

Pathlines Colored by Velocity Magnitude ( $m / s)$ (Time $=1.2000 e-01)$

(a) Case 1

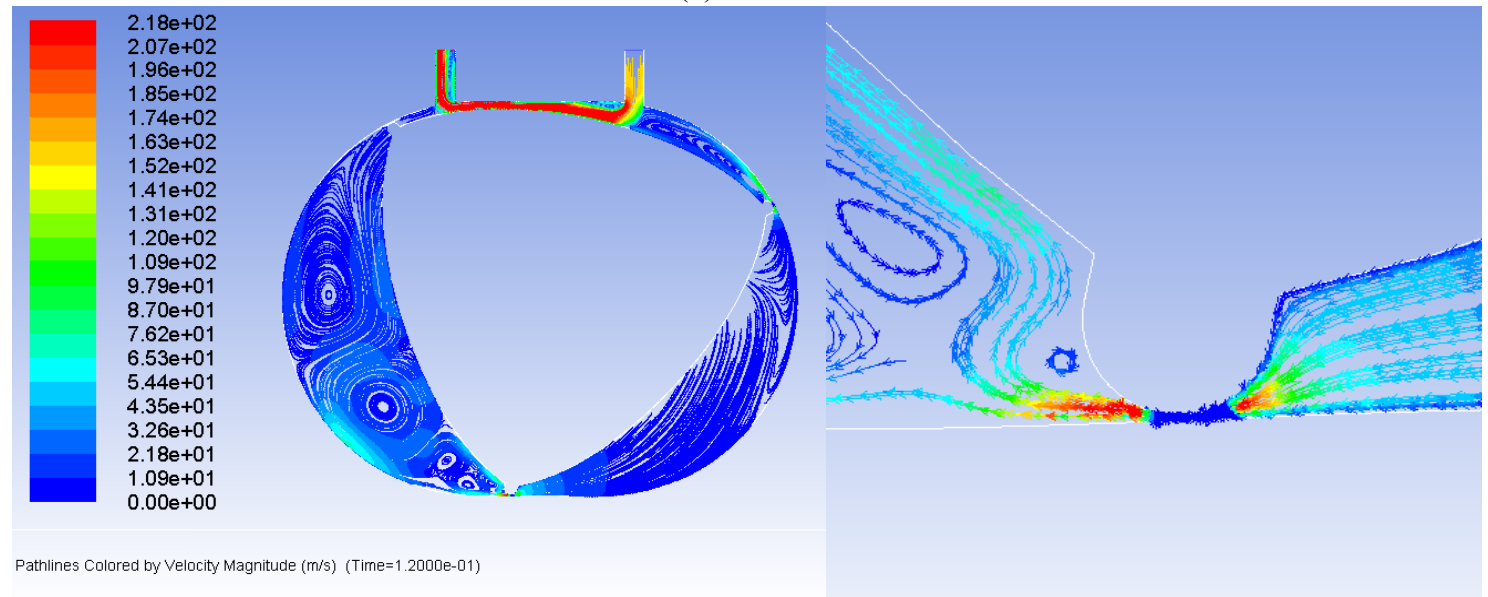

(b) Case 2

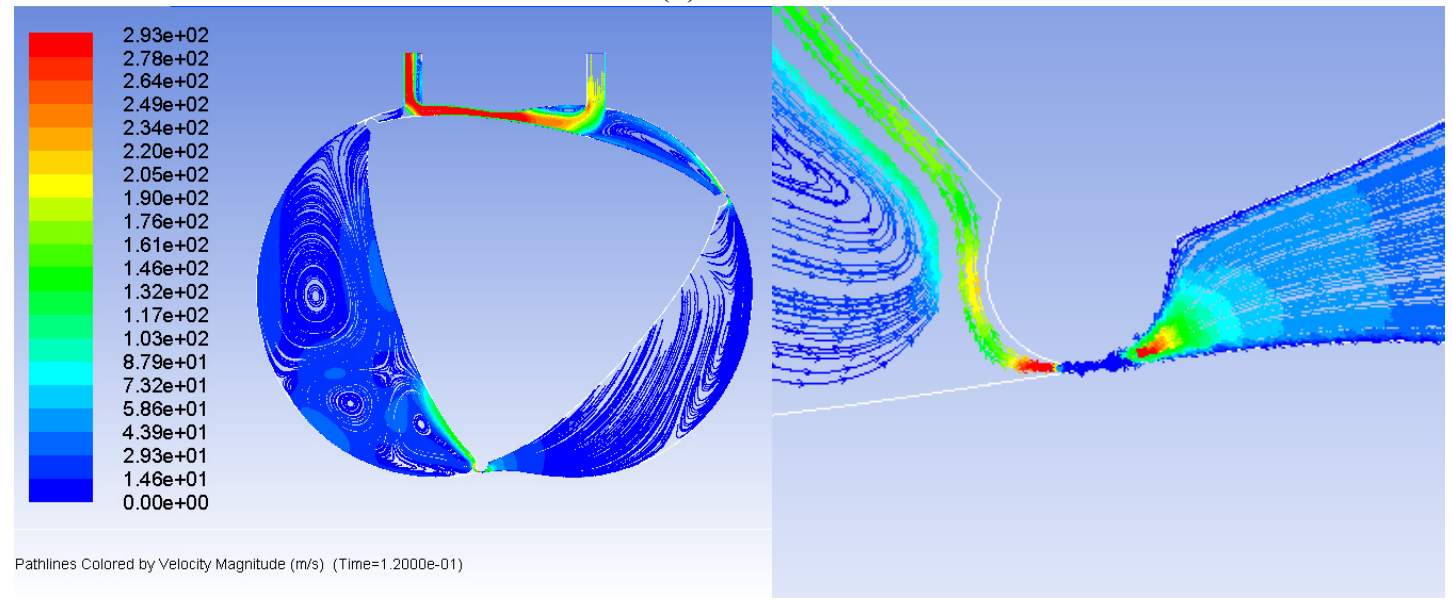

(c) Case 3

Figure 11 . Velocity plot in the three cases 


\section{Conclusions}

This study analyzes the effects on rotary engine flow characteristics of different design parameters; here, three variations in the curve $(K)$ factor. Within the three different curve designs, a larger working chamber volume and higher compression efficiency with a lower $K$ factor leads to higher leakage, larger internal pressure fluctuation, and unstable inlet pressure. Conversely, a high $\mathrm{K}$ factor produces low leakage, small internal pressure fluctuation, and stable inlet pressure, indicating a greater design advantage. Hence, when designing a rotary engine, designers must consider the interactions between profile curves and fluid flow characteristics. This paper can serve as a useful reference for such consideration.

\section{References}

Amrouche, F., Erickson, P., Park, J., \& Varnhagen, S. (2014). An experimental investigation of hydrogen enriched gasoline in a Wankel rotary engine. International Journal of Hydrogen Energy, 39, 8525-8534. http://10.1016/j.ijhydene.2014.03.172

Beard, J. E., \& Pennock, G. R. (1997). Force analysis of the apex seals in the Wankel rotary compressor including the influence of fluctuations in the crankshaft speed. Mechanism and Machine Theory, 32, 349-361. http://10.1016/S0094-114X(96)00051-1

Beard, J. E., Hall, A. S., \& Soedel, W. (1991). Comparison of hypotrochoidal and epitrochoidal gerotors. ASME J. Mech. Design, 113, 133-141. http://dx.doi.org/10.1115/1.2912761

Bonandrini, G., Mimmi, G., \& Rottenbacher, C. (2009). Theoretical analysis of internal epitrochoidal and hypotrochoidal machines. Proceedings of the Institution of Mechanical Engineers, Part C: Journal of Mechanical Engineering Science, 223, 1469-1480. http://dx.doi.org/10.1243/09544062JMES1163

Colbourne, J. R. (1974). The geometry of trochoid envelopes and their application in rotary pumps. Mechanism and Machine Theory, 9, 421- 435. http://dx.doi.org/10.1016/0094-114X(74)90025-1

Rose, S. W., \& Yang, D. C. H. (2013). Design of rotary engine from the apex seal profile (Abbr.: Rotary engine design by apex seal). Mechanism and Machine Theory, 64, 200-209. http://10.1016/j.mechmachtheory.2013. 01.015

Rose, S. W., \& Yang, D. C. H. (2014). The deviation function method of rotary engine design by geometric parameters. ASME J. Mech. Design, 136, 051004. http://10.1115/1.4026867

Rose, S. W., \& Yang, D. C. H. (2014). Wide and multiple apex seals for the rotary engine (Abbr.: Multi-apex-seals for the rotary engine). Mechanism and Machine Theory, 74, 202-215. http://10.1016/j.mechmachtheory.2013.12.011

Salanki, P. A., \& Wallace James, S. (1996). Evaluation of the hydrogen fuelled rotary engine for hybrid vehicle application. SAE paper, no. 960232. http://dx.doi.org/10.4271/960232

Shih, A. J. (1993). Analysis and comparison of epicycloidal and hypocycloidal internal combustion engine mechanisms. ASME J. Mech. Design, 115, 960-966. http://10.1115/1.2919294

Shih, A. J. (1993). Kinematics of the cycloidal internal combustion engine mechanism. ASME J. Mech. Design, 115, 953-959. http://10.1115/1.2919293

\section{Copyrights}

Copyright for this article is retained by the author(s), with first publication rights granted to the journal.

This is an open-access article distributed under the terms and conditions of the Creative Commons Attribution license (http://creativecommons.org/licenses/by/3.0/). 